Knowledge Exchange and Dissemination plan was to reengage with key stakeholders to present and request their feedback on trial findings, and to inform the design of a future definitive effectiveness trial.

Methods Stakeholders who supported recruitment of women and/or trial planning, representatives of the Irish Cancer Society, the Ireland's Health Service Executive, community organisations and pharmacies, GPs and primary care staff were invited to participate in an online interactive workshop. A Policy Brief which summarised trial findings was disseminated beforehand. Specific workshop objectives were to capture stakeholders' views on improvements to community engagement, strategies to enhance recruitment and retention, and policy and practice priorities arising from the research. Workshop participants were invited to fill in an anonymous, openended questionnaire after the workshop to register any further views on the previously discussed topics. Field notes taken during the workshop and questionnaire responses were combined to obtain a final list of challenges, barriers and recommendations for policy development and future research from a community perspective.

Results Forty-one stakeholders attended the workshop. The need for additional time to build relationships with local stakeholders for participant referral was identified. Further development of the social prescribing model in primary care was recommended as a vehicle to enhance recruitment. Low literacy was identified as a barrier to recruitment and retention, to be addressed by simplifying trial-related information, and by greater assistance with data completion. Other recommendations included provision of an intervention boost after trial completion to facilitate retention, and to maintain the established group support by encouraging participants to join other healthy community programmes. Key policy priorities were to remove cost and administrative barriers to access NRT, prioritise smoking cessation support tailored to disadvantaged groups, and to recognise and fund the peer-support model for smoking cessation.

Conclusion These results yielded important strategies to optimise the design of a future trial to assess WCQ effectiveness on smoking cessation for women smokers living in disadvantaged districts in Ireland. The findings may be generalisable to other community-based health interventions.

\section{P46 EVOLUTION OF GEOGRAPHICAL INEQUALITIES IN COVID-19 MORTALITY OVER THE FIRST WAVE OF THE PANDEMIC IN ENGLAND}

${ }^{1}$ Claire Welsh* ${ }^{*}$ 1,2 Viviana Albani, ${ }^{1,2}$ Fiona Matthews, ${ }^{1,2}$ Clare Bambra. ${ }^{1}$ Population Health Sciences Institute, Newcastle University, Newcastle, UK; ${ }^{2}$ Applied Research Collaboration North East and North Cumbria, Newcastle University, Newcastle, UK

\subsection{6/jech-2021-SSMabstracts. 134}

Background Early in the COVID-19 pandemic it was clear that inequalities were emerging in the distribution of deaths, with more deprived areas harder hit than less deprived ones. How these inequalities began, evolved, and changed with the implementation of the first national lockdown did not receive as much attention. We used COVID-19 death counts per local authority (LA) over the first wave (up to 4th July, 2020) to understand how COVID-19 impacted different areas, and to shed light on the equity effects of a strict national lockdown.
Methods Weekly COVID-19 death counts per LA were provided by the Office for National Statistics, along with population estimates for mid-2019 and data on the index of multiple deprivation (IMD) and median age per LA. Mortality rates for each area were calculated as deaths per 100,000 persons. Characteristics of the evolution of mortality rate per LA and decile of IMD were calculated, for example the speed of increase to each LAs peak rate. Simple linear models and descriptive statistics were used to compare areas.

Results More deprived LAs tended to begin recording COVID-19 deaths earlier than less deprived areas. Mortality rates in more deprived LAs rose at a faster rate to their peak, which was also higher than in less deprived LAs. All LA death rates peaked between 3 and 9 weeks following the implementation of the first lockdown. The time between the lockdown announcement and the peak death rate was significantly negatively associated with the speed of increase, adjusted for the median age of the population (95\% CI 0.33 to -0.31 deaths per 100,000 per week). Total cumulative mortality varied from 10.79 to 170.61 deaths per 100,000 persons per LA. Cumulative death rates were significantly higher in the most deprived $10 \%$ of LAs compared to others $(\mathrm{p}<0.001)$. Until lockdown, the most deprived $20 \%$ of LA's recorded $64 \%$ more deaths than the most deprived 20\%. By 4th July, this gap had narrowed to $16 \%$.

Conclusion Variation in the timing and speed of increase of local mortality rate curves occurred in the first wave and led to large inequalities across deprivation quintiles. This inequality was reduced but not completely abolished by the strict national lockdown imposed in March 2020. Timings of future national lockdowns should consider differences in local epidemic evolution, with extra support offered to those areas hardest hit.

\section{P47 INTERROGATING STRUCTURAL INEQUALITIES IN COVID- 19 MORTALITY IN ENGLAND AND WALES}

1,2 Gareth Griffith*, 1,2 George Davey Smith, ${ }^{4}$ David Manley, 1,2Laura Howe, ${ }^{3}$ Gwilym Owen. ${ }^{1}$ Medical Research Council Integrative Epidemiology Unit, University of Bristol, Bristol, UK; ${ }^{2}$ Population Health Sciences, Bristol Medical School, Bristol, UK; ${ }^{3}$ Department of Public Health and Policy, University of Liverpool, Liverpool, UK; ${ }^{4}$ School of Geographical Sciences, University of Bristol, Bristol, UK

\subsection{6/jech-2021-SSMabstracts. 135}

Background Numerous observational studies have highlighted structural inequalities in COVID-19 mortality in the UK. Such studies often fail to consider the complex spatial nature of such inequalities in their analysis, leading to the potential for bias and an inability to reach conclusions about the most appropriate structural levels for policy intervention.

Methods We use publicly available population data on COVID-19 related- and all-cause mortality between March and July 2020 in England and Wales to investigate the spatial scale of such inequalities. We propose a multiscale approach to simultaneously consider four spatial scales at which processes driving inequality may act and apportion inequality between these.

Results Adjusting for population age structure, number of care homes and residing in the North we find highest regional inequality in March and June/July. We find finer-grained within-region increased steadily from March until July. The 
importance of spatial context increases over the study period. No analogous pattern is visible for non-COVID mortality. Higher relative deprivation is associated with increased COVID-19 mortality at all stages of the pandemic but does not explain structural inequalities.

Conclusion Results support initial stochastic viral introduction in the South, with initially high inequality decreasing before the establishment of regional trends by June and July, prior to reported regionality of the 'second-wave'. We outline how this framework can help identify structural factors driving such processes, and offer suggestions for a long-term, locally-targeted model of pandemic relief in tandem with regional support to buffer the social context of the area.

\section{P48 RISK OF COVID-19 INFECTION AMONG MEN AND WOMEN DURING THE LOCKDOWN OF SPRING 2020 IN FRANCE}

${ }^{1}$ Lola Neufcourt*, ${ }^{1}$ Camille Joannès, ${ }^{1}$ Marine Maurel, ${ }^{1}$ Niamh $M$ Redmond, ${ }^{1}$ Cyrille Delpierre, ${ }^{1,2}$ Michelle Kelly-Irving. ' CERPOP, University, de Toulouse, Inserm, UPS, Toulouse, France, ${ }^{2}$ Iferiss-Fed 4241, University, Toulouse III Paul Sabatier, Toulouse, France

\subsection{6/jech-2021-SSMabstracts. 136}

Background In the context of the Covid-19 pandemic, several factors such as age, chronic disease or obesity have been associated with adverse outcomes and mortality from Covid-19. However, the social distribution of Covid-19 infection among men and women was largely neglected in France, mainly due to a lack of data. The aim of this study is to describe and analyse the risk of Covid-19 infection in relation to sex, and the influence of other social factors, specifically occupation, in this association.

Methods We used data from the citizen science initiative 'Baromètre Covid-19'. Each week, an internet survey was administered to a sample of 5,000 people representative of the French mainland population aged 18 and over, using the quota method. A total of 25,001 participants were interviewed between 7 April and 11 May 2020. We used multivariable nested logistic regression modelling to study the relationship between sex, occupation and Covid-19 infection. Confounders included age, region of residence, population density, whether you worked outside of home during the lockdown, house overcrowding, comorbidities and body mass index.

Results Women reported a medical diagnosis of Covid-19 infection more often than men (4\% vs. $3.2 \%)$. In a model adjusted for confounders, women were $23 \%$ more likely to report a medical diagnosis of Covid-19 infection than men $(\mathrm{OR}=1.23$ [95\%-CI=1.06-1.42]). Controlling for sex and socioeconomic variables (occupation), the risk of infection for women was reversed $(\mathrm{OR}=0.84[95 \%-\mathrm{CI}=0.59-1.19])$. While most men, other than executives, were less likely to report the infection, this association was not observed amongst women.

Conclusion Occupation was found to influence the relationship between sex and Covid-19 infection suggesting a gender effect. The differences in the risk of infection between men and women require exploration with regard to socioeconomic factors. The social roles of women and men are associated with a non-random distribution of the virus, potentially reflecting structural societal inequalities.

\section{P49 COVID-19 AT THE DEEP END: EXPERIENCES OF PRIMARY CARE STAFF WORKING IN THE MOST DEPRIVED AREAS OF ENGLAND DURING THE COVID-19 PANDEMIC}

Claire Norman*, Josephine M Wildman, Sarah Sowden. Population Health Sciences Institute, Newcastle University, Newcastle upon Tyne, UK

\subsection{6/jech-2021-SSMabstracts. 137}

Background COVID-19 is disproportionately impacting people in low-income communities. Primary care staff in areas of high blanket deprivation (also known as the 'Deep End') have unique insights into the challenges posed by the pandemic. We aim to explore the impact of the COVID-19 pandemic from the perspective of Deep End primary care practitioners in North East England, the most deprived region of the country.

Methods Semi-structured interviews followed by thematic analysis. 13 participants were interviewed (11 GPs, 1 nurse practitioner and 1 district nurse) with Deep End careers ranging from 3 months to 31 years. Participants were recruited via purposive and snowball sampling. Semi-structured interviews were conducted using video-conferencing software. Data were analysed using thematic content analysis. Participants were interviewed between September-December 2020, at the start of the UK second wave of the COVID-19 pandemic.

Results Our results can be categorised into three broad themes: the immediate health risks of COVID-19 on Deep End general practices and patients; factors likely to exacerbate the effects of existing socioeconomic deprivation; and wider implications for remote consulting.

Discussion Deep End practitioners have valuable insights into the impact of social distancing restrictions and remote consulting on patients' health and wellbeing and on the delivery of primary care in areas of deep deprivation. Their experiences should guide future pandemic response measures and any move to 'digital first' primary care to ensure that existing inequalities are not worsened.

\section{P50 TIME-VARYING SELECTION BIAS IN ANALYSES OF COVID-19 IN UK BIOBANK}

Alice R Carter*, Gareth J Griffith, Apostolos Gkatzionis, Rachael Hughes, George Davey Smith, Deborah Lawlor, Kate Tilling. Medical Research Council Integrative Epidemiology Unit, Population Health Sciences, University of Bristol, Bristol, UK

\subsection{6/jech-2021-SSMabstracts. 138}

Rationale Associations between COVID-19 risk factors and COVID-19 outcomes change over time, likely due to selection into who receives a COVID-19 test. When studies do not account for the changes in testing criteria, the association between a risk factor and outcome is a joint estimate across time. The transportability of a joint estimate aggregated over multiple testing periods may be limited. To improve generalisability, it is desirable to estimate effects net of time-varying selection.

Aim 1) Demonstrate variation in the association between covariates expected to associate with testing, and those which would not, on COVID-19 at different timepoints. 2) Apply methods to mitigate biases in empirical estimates.

Methods Analyses will be carried out on up to 421,037 UK Biobank participants residing in England at baseline (mean 